\title{
Bargaining in Dynamic Games
}

\author{
Leon A. Petrosyan \\ St. Petersburg University \\ $26^{\text {th }}$ European Conference on Operational Research
}

Rome 1-4 July, 2013 


\section{Classical control problem.}

$$
\begin{aligned}
& \dot{x}=f(x, u), \quad x \in R^{n}, u \in U \subset \operatorname{CompR}^{\prime}, \\
& x\left(t_{0}\right)=x_{0}, \quad t \in\left[t_{0}, T\right], \\
& H(x(T))=-\rho(x(T), M) .
\end{aligned}
$$

$C\left(x_{0}, T-t_{0}\right)$ - reachability set.

$\bar{x}(t)$ - optimal trajectory.

$\Gamma\left(x_{0}, T-t_{0}\right), \quad \Gamma(\bar{x}(t), T-t), \quad C(\bar{x}(t), T-t)$

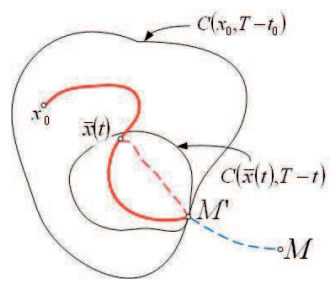

R. Bellmann

Time-consistency, Strong Time-consistency. 


\section{Multycriterial control.}

$$
\begin{aligned}
& \dot{x}=f(x, u), \quad x \in R^{n}, u \in U \subset \operatorname{CompR}^{\prime}, \\
& x\left(t_{0}\right)=x_{0}, \quad t \in\left[t_{0}, T\right], \\
& H(x(T))=\left\{H_{1}(x(T)), \ldots, H_{k}(x(T))\right\} .
\end{aligned}
$$

Let $k=3, H_{i}(x(T))=-\rho\left(x(T), M_{i}\right)$.

Pareto-optimal solution.

$\bar{x}(t)$ - Pareto-optimal trajectory.

$\Gamma\left(x_{0}, T-t_{0}\right), \quad \Gamma(\bar{x}(t), T-t), \quad C\left(x_{0}, T-t_{0}\right), \quad C(\bar{x}(t), T-t)$, $P\left(x_{0}, T-t_{0}\right), P(\bar{x}(t), T-t)$

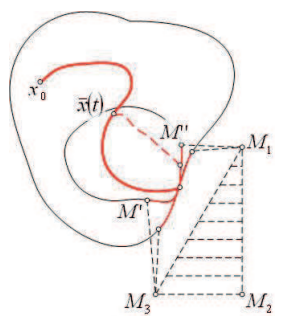




\section{Nash bargaining solution in Differential Games.}

$$
\begin{aligned}
& \dot{x}=f\left(x, u_{1}, \ldots, u_{n}\right), \quad x \in R^{m}, u_{i} \in U_{i} \subset C \text { CompR } R^{\prime}, \\
& x\left(t_{0}\right)=x_{0}, \quad t \in\left[t_{0}, T\right],
\end{aligned}
$$

The payoff of player $i \rightarrow H_{i}(x(T))$,

$\Gamma\left(x_{0}, T-t_{0}\right)$

$W\left(x_{0}, T-t_{0} ;\{i\}\right)$ - the garanteed payoff of player $i$

NB.

$\max _{x^{\prime} \in C\left(x_{0}, T-t_{0}\right)} \prod_{i=1}^{n}\left(H_{i}\left(x^{\prime}\right)-W\left(x_{0}, T-t_{0} ;\{i\}\right)\right)=\prod_{i=1}^{n}\left(H_{i}(\overline{\bar{x}})-W\left(x_{0}, T-t_{0} ;\{i\}\right)\right)$

$\bar{x}(t), \quad x_{0} \rightarrow \overline{\bar{x}}, \quad \Gamma(\bar{x}(t), T-t), t \in\left[t_{0}, T-t_{0}\right], \quad W(\bar{x}(t), T-t ;\{i\})$

$\max _{x^{\prime} \in C(\bar{x}(t), T-t)} \prod_{i=1}^{n}\left(H_{i}\left(x^{\prime}\right)-W(\bar{x}(t), T-t ;\{i\})\right)=\prod_{i=1}^{n}\left(H_{i}(\overline{\bar{x}}(\bar{x}(t)))-W(\bar{x}(t), T-t ;\{i\})\right)$

$\overline{\bar{x}}(\bar{x}(t)) \neq$ const $\neq \bar{x}$

NB, not TC, not STC 
Regularization. Here we consider simple case of the game with terminal payoff and prescribed duration.

Denote the payoff function as

$$
K_{i}\left(x_{0}, T-t_{0} ; u_{1}(\cdot), \ldots, u_{n}(\cdot)\right)=H_{i}(x(T)),
$$

where $x(t)$ is the solution of problem

$$
\begin{gathered}
\dot{x}=f\left(x, u_{1}, \ldots, u_{n}\right) \\
x\left(t_{0}\right)=x_{0}
\end{gathered}
$$

Denote the corresponding differential game by $\Gamma\left(x_{0}, T-t_{0}\right)$. Consider a Bargaining Problem for each subgame $\Gamma(y, T-t)$, where $y \in C\left(x_{0}, t\right)$, and $C\left(x_{0}, t\right)$ is the reachability set for system (1). We can have in mind as bargaining solution Nash Bargaining (NB), (KS). Suppose we define for each $y \in C\left(x_{0}, t\right)$, $t \in\left[t_{0}, T\right]$ the NB solution and denote it by $D(y, T-t)$.

$$
D(y, T-t)=\left\{H_{i}(\widetilde{x}(T ; y, t))\right\}
$$


Suppose $\widetilde{x}(t), t \in\left[t_{0}, T\right]$ is the bargain trajectory in the game $\Gamma\left(x_{0}, T-t_{0}\right)$ leading from initial point $x_{0}$ to bargaining point $\widetilde{x}\left(T ; x_{0}, t_{0}\right)$. As we mention the NB solution as many other bargaing solutions (including Kalai-Smorodinsky) are time inconsistent, which means that

$$
D(\widetilde{x}(t), T-t) \neq \text { const } \quad t \in\left[t_{0}, T\right] .
$$

This makes difficult the implementation of NB in practice.

Here we purpose the regularization mechanism similar to IDP we used considering the similar time-inconsistency problem in differential cooperative games. To look different from IDP (imputation distribution procedure) we shall call in PDP (payoff distribution procedure). 
Introduce the function $g_{i}(\tau), \tau \in\left[t_{0}, T\right]$ such that

$$
D_{i}\left(x_{0}, T-t_{0}\right)=H\left(\widetilde{x}\left(T ; x_{0}, T-t_{0}\right)\right)=\int_{t_{0}}^{T} g_{i}(\tau) d \tau .
$$

$g_{i}(\tau)$ is a new control variable $\left(\tau \in\left[t_{0}, T\right]\right)$ used to distribute the payoff over the time interval $\left[t_{0}, T\right]$.

Time-consistency condition

$$
D_{i}\left(x_{0}, T-t_{0}\right)=\int_{t_{0}}^{t} g_{i}(\tau) d \tau+D_{i}(\widetilde{x}(\tau), T-\tau) .
$$

If function $D_{i}$ is differentiable we get

$$
g_{i}(t)=-\frac{d}{d t} D_{i}(\widetilde{x}(\tau), T-t),
$$

or

$$
g_{i}(t)=-\sum_{i=1}^{n} \frac{\partial D_{i}}{\partial x_{i}} f_{i}\left(\widetilde{x}(t), \widetilde{u}_{1}(t), \ldots, \widetilde{u}_{n}(t)\right), \quad i=1, \ldots, n .
$$


Kalai-Smorodinsky solution

$$
\begin{gathered}
\dot{x}=f\left(x, u_{1}, \ldots, u_{n}\right), \quad x \in R^{m}, u_{i} \in U \subset \operatorname{Comp} R^{\prime} \\
x\left(t_{0}\right)=x_{0}, \quad t \in\left[t_{0}, T\right] .
\end{gathered}
$$

The payoff of player $i-H_{i}(x(T))$

$\Gamma\left(x_{0}, T-t_{0}\right)$

$W\left(x_{0}, T-t_{0} ;\{i\}\right)$ - the max guaranteed payoff of player $i$

KS-solution

Consider the set $\aleph\left(x_{0}, T-t_{0}\right)$ of all possible payoffs of players.

$$
\aleph\left(x_{0}, T-t_{0}\right)=\left\{H_{1}(x(T)), \ldots, H_{n}(x(T))\right\}
$$

for all possible $x(T) \in C\left(x_{0}, T-t_{0}\right)$ where $C\left(x_{0}, T-t_{0}\right)$ is the reachability set of (1).

Correspondingly denote by $\aleph(y, T-t), y \in C\left(x_{0}, T-t_{0}\right)$ the set of all possible payoffs of players in subgames starting from $y$ with duration $T-t(\Gamma(y, T-t))$. Denote by

$$
\bar{H}_{i}=\max _{x(T) \in C\left(x_{0}, T-t_{0}\right)} H(x(T)) .
$$

Consider a line $L$ connecting the "status point" $W$ with $\bar{H}$. 
The intersection of $L$ and $\aleph\left(x_{0}, T-t_{0}\right)$ is called Kalai-Smorodinsky solution (KS-solution).

Example. KS-solution is time-inconsistent

$$
\begin{gathered}
\dot{x}=u_{1}+u_{2}, \quad\left|u_{1}\right| \leq 1,\left|u_{2}\right| \leq 1, x \in R^{2}, u_{1}, u_{2} \in R^{2} \\
x_{0}=(6,3), t \in[0,2], x=\left(x_{1}, x_{2}\right) \\
H_{i}\left(x_{0}, 2 ; u_{1}, u_{2}\right)=-x(2), K_{2}\left(z_{0}, 2 ; u_{1}, u_{2}\right)=-|y(2)|
\end{gathered}
$$

Problem $\Gamma\left(x_{0}, 2\right)$

$W\left(x_{0}, 2\right)=(-6,3)$

$\bar{H}\left(x_{0}, 2\right)=(-2,0)$

and corresponds to the point $\widehat{x}\left(x_{0}, 2\right)=(2,0)$, since $\max H_{1}=-2$, $\max H_{2}=0$. $C^{2}(6,3 ; 2)$ is circle with center $(6,3)$ and radius 4.

Optimal trajectory is to move from $x_{0}=(6,3)$ to $\widehat{x}\left(x_{0}, 2\right)=(2,0)$ with maximal velocity till the intersection with $C(6,3 ; 2)$. The intersection point is $\mathrm{KS}$-solution of $\Gamma\left(x_{0}, 2\right)$. 
We see that when moving along $\mathrm{KS}$-solution trajectory $\bar{x}(t)$ connecting $x_{0}$ with $\mathrm{KS}$-solution of the problem $\Gamma\left(x_{0}, 2\right)$ in subgame $\Gamma(\bar{x}(1), 1)$ we get a new $\mathrm{KS}-$ solution which do not coincide with previous $\mathrm{KS}$-solution of the game $\Gamma\left(x_{0}, 2\right)$.

$$
K S\left(x_{0}, 2\right) \neq K S(\bar{x}(1), 1)
$$

(time-inconsistency)

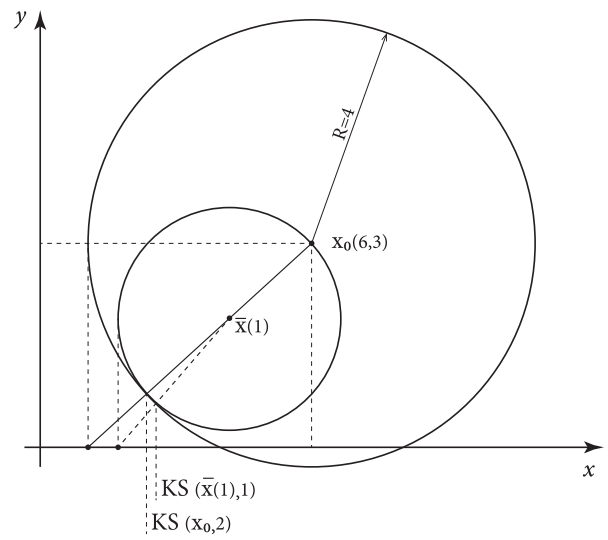




\section{Differential Cooperative Game.}

Differential Cooperative Game $\Gamma\left(x_{0}, T-t_{0}\right)$ with prescribed duration $T-t_{0}$ from the initial position $x_{0}$.

$$
\dot{x}=f\left(x, u_{1}, \ldots, u_{n}\right), \quad x \in R^{n}, u_{i} \in U_{i}
$$

integral payoff

$$
K_{i}\left(x_{0}, T-t_{0} ; u_{1}, \ldots, u_{n}\right)=\int_{t_{0}}^{T} h_{i}(x(t)) d t, \quad h_{i}>0, i=1, \ldots, n .
$$

Cooperative form of $\Gamma\left(x_{0}, T-t_{0}\right)$.

Cooperative behavior $u^{*}(t)=\left\{u_{1}^{*}(t), \ldots, u_{n}^{*}(t)\right\}$

$$
\begin{aligned}
\sum_{i=1}^{n} K_{i}\left(x_{0}, T-t_{0} ; u_{1}^{*}, \ldots, u_{n}^{*}\right) & = \\
& =\max _{u_{1}, \ldots, u_{n}} \sum_{i=1}^{n} K_{i}\left(x_{0}, T-t_{0} ; u_{1}, \ldots, u_{n}\right)= \\
& =\sum_{i=1}^{n} \int_{t_{0}}^{T} h_{i}\left(x^{*}(t)\right) d t=v\left(N ; x_{0}, T-t_{0}\right),
\end{aligned}
$$

$x^{*}(t)$ - cooperative trajectory. 
Characteristic Function in $\Gamma\left(x_{0}, T-t_{0}\right)$. $v\left(S ; x_{0}, T-t_{0}\right), \quad S \subset N$, superadditivity: $v\left(S_{1} \cup S_{2} ; x_{0}, T-t_{0}\right) \geq v\left(S_{1} ; x_{0}, T-t_{0}\right)+v\left(S_{2} ; x_{0}, T-t_{0}\right)$, $S_{1} \cap S_{2}=\emptyset$.

There are different ways on how to define $c . f$.

a. Classical: $v\left(S ; x_{0}, T-t_{0}\right)=V a / \Gamma_{S, N \backslash S}\left(x_{0}, T-t_{0}\right)$, where $\Gamma_{S, N \backslash S}\left(x_{0}, T-t_{0}\right)$ is a zero-sum game played upon the structure of game $\Gamma\left(x_{0}, T-t_{0}\right)$ between $S$ as player 1 and $N \backslash S$ as player 2 .

b. $v\left(S ; x_{0}, T-t_{0}\right)=\sum_{i \in S} K_{i}\left(x_{0}, T-t_{0} ; \bar{u}_{S}, \bar{u}_{N \backslash S}\right)$, where $\left(\bar{u}_{S}, \bar{u}_{N \backslash S}\right)$ is some giver NE in $\Gamma_{S, N \backslash S}^{\prime}$ played as non zero-sum game over the structure of $\Gamma\left(x_{0}, T-t_{0}\right)$ between two players: coalition $S$ as player 1 and $N \backslash S$ as player 2

c. $v\left(S ; x_{0}, T-t_{0}\right)=\max _{u_{S}=\left\{u_{i}, i \in S\right\}} \sum_{i \in S} K_{i}\left(x_{0}, T-t_{0} ; \bar{u} \| u_{S}\right)$, where $\bar{u}=\left(\bar{u}_{1}, \ldots, \bar{u}_{n}\right)$ is some fixed NE in $\Gamma\left(x_{0}, T-t_{0}\right)$.

L. Petrosjan, G. Zaccour Time-consistent Shapley value allocation of pollution cost reduction // Journal of Economics Dunamics \& Control, 27 (2003), pp. 381-398. 
Let $E\left(x_{0}, T-t_{0}\right)$ be the imputation set in $\Gamma\left(x_{0}, T-t_{0}\right)$ :

$E\left(x_{0}, T-t_{0}\right)=\left\{\xi=\left(\xi_{i}\right): \sum_{i=1}^{n} \xi_{i}=v\left(N ; x_{0}, T-t_{0}\right), \xi_{i} \geq v\left(\{i\} ; x_{0}, T-t_{0}\right), i \in N\right\}$.

Denote by $C^{t-t_{0}}\left(x_{0}\right), t \in\left[t_{0}, T\right]$ reachable set of the (1).

For each $y \in C^{t-t_{0}}\left(x_{0}\right)$ consider a subgame $\Gamma(y, T-t)$ of the game $\Gamma\left(x_{0}, T-t_{0}\right)$, with corresponding c. f. $v(S ; y, T-t)$ and set of imputations $E(y, T-t)$.

Definition. A point-to-set mapping $C(y, T-t) \subset E(y, T-t)$ defined for all $y \in C^{t-t_{0}}\left(x_{0}\right), t \in\left[t_{0}, T\right]$ is call solution concept (SC) in the family of subgames $\Gamma(y, T-t)$.

In special cases $C(y, T-t)$ may be a core, NM-solution, Shapley value, nucleous etc.

What happens when the game develops along the cooperative trajectory $x^{*}(t)$ ? We pass through current subgame $\Gamma\left(x^{*}(t), T-t\right)$, wilingly or not updating the current $\mathrm{SC} \leftrightarrow C\left(x^{*}(t), T-t\right)$. 
Imputation Distribution Procedure (IDP).

Let $\bar{\xi} \in C\left(x_{0}, T-t_{0}\right)$ and $\beta_{i}(t), i \in N, t \in\left[t_{0}, T\right]$ satisfies the condition

$$
\bar{\xi}=\int_{t_{0}}^{T} \beta_{i}(t) d t, \quad i \in N, \quad \beta_{i} \geq 0 .
$$

$\beta_{i}(t)$ is called IDP.

Define

$$
\bar{\xi}(\theta)=\int_{t_{0}}^{\theta} \beta_{i}(t) d t, \quad i \in N, \quad \beta_{i} \geq 0 .
$$

Definition. The SC $C\left(x^{*}(t), T-t\right), t \in\left[t_{0}, T\right]$ is called time-consistent (TC) if there exist such IDP $\beta(t)=\left\{\beta_{i}(t)\right\}$ that

$$
\bar{\xi}-\bar{\xi}(\theta) \in C\left(x^{*}(\theta), T-\theta\right)
$$

for all $\theta \in\left[t_{0}, T\right]$.

Definition. The SC $C\left(x^{*}(t), T-t\right), t \in\left[t_{0}, T\right]$ is called strongly time-consistent (STC) if there exist such IDP $\beta(t)=\left\{\beta_{i}(t)\right\}$ that

$$
\bar{\xi}(\theta) \oplus \bar{C}\left(x^{*}(\theta), T-\theta\right) \subset C\left(x_{0}, T-t_{0}\right)
$$

for all $\theta \in\left[t_{0}, T\right]$. Here $\bar{\xi} \oplus A$ means the set of all possible vectors $\bar{\xi}+\eta$ for all $\eta \in A$. 
Consider $C\left(x^{*}(t), T-t\right)$ along $x^{*}(t), t \in\left[t_{0}, T\right]$. Suppose we can construct a differentiable selector $\xi^{t} \in C\left(x^{*}(t), T-t\right)$, then we can easily get for $\beta(t)$ the following formula

$$
\begin{gathered}
\bar{\xi}=\bar{\xi}(\theta)+\xi^{t} \rightarrow \bar{\xi}=\int_{t_{0}}^{\theta} \beta_{i}(t) d t+\xi^{t} \\
\beta_{i}(t)=-\frac{d}{d t} \xi^{t}
\end{gathered}
$$

If $\xi^{t}$ can be chosen as monotonic nonincreasing (which is very possible since $h_{i}>0$, then $\beta_{i} \geq 0$, and SC is TC.

If the case (for instance) $C(y, T-t)$ is a Shapley value, we get

$$
\begin{gathered}
\beta_{i}(t)=-\sum_{S \subset N, S \ni i} \frac{(n-s) !(s-1) !}{n !}\left[\frac{d}{d t} v\left(x^{*}(t), T-t ; S\right)-\right. \\
\left.-\frac{d}{d t} v\left(x^{*}(t), T-t ; S \backslash\{i\}\right)\right]
\end{gathered}
$$

and we need only differentiability of the value function (c. f.) $v(x, T-t ; S)$. 


\section{References}

Neumann J., Morgenstern O. (1947). "Theory of Games and Economic Behavior Princeton.

Nash J. (1951). "Non-cooperative games Ann.

Mathematics, 54, pp. 286-295.

Petrosjan L. A. (1993). "Differential Games of Pursuit World Scientific, Singapore.

Petrosyan L. A., Yeung D. W. K. (2006) Cooperative Stochastic Differential Games. Springer, 242 p.

Shapley L.S. (1953). "A Value for $n$-Person Games Contributions to the Theory of Games (in H.W. Kuhn and A.W. Tucker (eds.), Princeton, Princeton University Press, pp. 307-315. 